\title{
Detection of Endogenous Retinoids in the Molluscan CNS and Characterization of the Trophic and Tropic Actions of 9-cis Retinoic Acid on Isolated Neurons
}

\author{
Jennifer M. Dmetrichuk, ${ }^{1}$ Robert L. Carlone, ${ }^{1}$ Timothy R. B. Jones, ${ }^{2}$ Nicholas D. Vesprini, ${ }^{1}$ and Gaynor E. Spencer ${ }^{1}$ \\ Departments of ${ }^{1}$ Biological Sciences and ${ }^{2}$ Chemistry, Brock University, St. Catharines, Ontario, Canada L2S 3A1
}

\begin{abstract}
Retinoic acid (RA) is an active metabolite of Vitamin A that plays an important role in the growth and differentiation of many cell types. All-trans RA (atRA) is the retinoic acid isomer that has been most widely studied in the nervous system, and can induce and direct neurite outgrowth from both vertebrate and invertebrate preparations. The presence and role of the 9-cis-RA isomer in the nervous system is far less well defined. Here, we used high-pressure liquid chromatography (HPLC) and mass spectrometry (MS) to show for the first time, the presence of both atRA and 9-cis-RA in the CNS of an invertebrate. We then demonstrated that 9-cis-RA was capable of exerting the same neurotrophic and chemotropic effects on cultured neurons as atRA. In this study, significantly more cells showed neurite outgrowth in 9-cis-RA versus the EtOH vehicle control, and 9-cis-RA significantly increased the number and length of neurites from identified neurons after $4 \mathrm{~d}$ in culture. 9-cis-RA also extended the duration of time that cells remained electrically excitable in culture. Furthermore, we showed for the first time in any species, that exogenous application of 9-cis-RA induced positive growth cone turning of cultured neurons. This study provides the first evidence for the presence of both atRA and 9-cis-RA in an invertebrate CNS and also provides the first direct evidence for a potential physiological role for 9-cis-RA in neuronal regeneration and axon pathfinding.
\end{abstract}

Key words: growth cone; retinoic acid; endogenous retinoid; 9-cis retinoic acid; Lymnaea stagnalis; cell culture

\section{Introduction}

The vitamin A metabolite, retinoic acid (RA), plays an essential role in development and regeneration of many cell types. During vertebrate development, RA is a key regulator of axis specification, cellular differentiation and patterning (Maden and Hind, 2003, Maden 2007). In the nervous system, RA induces axonal outgrowth from embryonic neurons of the spinal cord and neural tube (Wuarin et al., 1990; Maden et al., 1998a), dorsal root ganglion (DRG) (Haskell et al., 1987; Corcoran et al., 2000), cerebellum (Yamamoto et al., 1996), and sympathetic ganglion (Plum et al., 2001). RA can also promote axonal outgrowth from differentiated spinal cord neurons from adult newts (Prince and Carlone, 2003; Dmetrichuk et al., 2005) and with other neurotrophins, from differentiated retinal ganglion cells (Mey and Rombach, 1999). Similar to other neural inducing factors (McFarlane and

\footnotetext{
Received July 9, 2008; revised 0ct. 3, 2008; accepted 0ct. 24, 2008.

This work was supported by a grant from the Natural Sciences and Engineering Research Council of Canada (NSERC) to G.E.S. and a Brock University, Canadian Institutes of Health Research Development Award to R.L.C. J.M.D. was supported by a post-graduate scholarship from NSERC (Canada). We are grateful to Dr. Michael Wagner (Department of Anatomy and (ell Biology, State University of New York) for his kind gift of the reporter cell line used in this study. For statistical guidance, we are thankful to Dr. Jean Richardson of the Biology Department at Brock University. We are grateful to Stephan Ohnmacht of the Chemistry Department at Brock University for his assistance and expertise with the NMR and UV-vis data. We also thank the Department of Chemistry (Brock University) for their collaboration and use of analytical equipment (LC/MS, NMR). We are also grateful to Dr. Vincent De Luca (Brock University) for the use of the UV-vis spectrometer.

Correspondence should be addressed to Gaynor E. Spencer, Department of Biological Sciences, Brock University, St. Catharines, Ontario, Canada L2S 3A1. E-mail: gspencer@brocku.ca.

DOI:10.1523/JNEUROSCI.3192-08.2008

Copyright $\odot 2008$ Society for Neuroscience $\quad$ 0270-6474/08/2813014-11 $\$ 15.00 / 0$
}

Holt, 1997), RA is also capable of directing neurite outgrowth (Maden et al., 1998a; Dmetrichuk et al., 2005, 2006).

A number of different isomers of RA exist, including all-trans RA (atRA) and 9-cis-RA. atRA has been identified in embryonic and adult nervous systems in many vertebrates (Maden et al., 1998b; Hoover et al., 2001; Werner and DeLuca, 2002), but in vivo plasma levels of 9-cis-RA are usually much lower than atRA (Malik et al., 2000) and often fall below detection limits (Kraft and Juchau, 1993). 9-cis-RA has, however, been detected in the neonatal rat eye and human embryonic brain (Kraft and Juchau, 1993) as well as during gastrulation in Xenopus (Kraft et al., 1994). 9-cis-RA has also been detected in the wound epidermis of regenerating limb blastemas of urodele amphibians and in fiddler crab limb blastemas, suggesting a role for 9-cis-RA in limb regeneration (Viviano et al., 1995; Hopkins, 2001). Despite its detection in some species, the mechanism for 9-cis-RA biosynthesis has not been determined, and whether it is isomerized from atRA or synthesized independently of atRA, is not yet known (Maden and Hind, 2003; Brodeur et al., 2007). It is also not clear whether atRA and 9-cis-RA have similar physiological roles. In differentiating human neuroblastoma cells, both 9-cis-RA and atRA produced a dose-dependent increase in neurite outgrowth and inhibition of proliferation (Han et al., 1995). 9-cis-RA, like atRA, is also a potent inducer of digit pattern duplications in the chick wing bud (Thaller et al., 1993). Very little information is available regarding the role of 9-cis-RA in the nervous system.

Using adult invertebrate neurons of Lymnaea stagnalis, we recently demonstrated that atRA induces neurite outgrowth and 
maintains electrical excitability of cultured neurons, in the absence of other neurotrophic factors. We also provided the first evidence of atRA-induced attractive turning of individual growth cones in vitro (Dmetrichuk et al., 2006). The physiological relevance of these findings was previously unclear, as retinoid detection in invertebrates has been limited. The studies reported here aimed to determine whether atRA and its geometric isomer, 9-cis-RA, are present in the CNS of the adult snail, Lymnaea stagnalis. We also aimed to determine, for the first time, whether 9-cis-RA exerts similar neurotrophic and/or neurotropic effects as atRA on cultured neurons.

\section{Materials and Methods}

Chemicals. High-pressure liquid chromatography (HPLC)-grade chemicals including acetonitrile, methanol and formic acid were obtained from Sigma-Aldrich. Milli-Q water was produced in-house with a resistivity of $18.2 \mathrm{M} \Omega / \mathrm{cm}$. AtRA, 9-cis-RA, cadmium, retinaldehyde, ethyl acetate, methyl acetate and butylated hydroxytoluene were all obtained from Sigma-Aldrich.

Retinal dehydrogenases assay. As an indirect measure of endogenous RA, retinal dehydrogenase (RALDH) activity was determined in Lymnaea tissue extracts and reaction products were then added to Sil-15 retinoid reporter cells (Wagner et al., 1992). Briefly, adult Lymnaea CNSs (18 pooled CNSs in 2 samples) were isolated in the dark and immediately frozen in liquid nitrogen. Samples were then freeze/thawed in a $37^{\circ} \mathrm{C}$ water bath/liquid nitrogen and homogenized in $250 \mu \mathrm{l}$ of homogenization buffer (10 mM PBS, pH 7.2, 1 mm phenylmethyl sulfonyl fluoride, 10 $\mu \mathrm{g} / \mathrm{ml}$ aprotinin). The homogenates were vortexed, spun for $12 \mathrm{~min}$ $(16,000 \mathrm{rpm})$ and the supernatants were removed and frozen at $-80^{\circ} \mathrm{C}$. A total of $20 \mu \mathrm{l}$ was set aside for total protein determination (Coomassie Blue dye-binding assay, Bio-Rad).

The equivalent volume from each supernatant, containing $4 \mu \mathrm{g}$ of protein, was added to eppendorf tubes and incubated at $37^{\circ} \mathrm{C}$ for $2 \mathrm{~h}$ with the following reagents; $10^{-7} \mathrm{M}$ retinaldehyde, $4 \mathrm{~mm}$ dithiothreitol, and $2.4 \mathrm{~mm}$ nicotinamide-adenine dinucleotide. Aliquots of each reaction mix were then diluted with culture medium [DMEM (Invitrogen-BRL), $10 \%$ fetal calf serum and $0.4 \mathrm{mg} / \mathrm{ml}$ geneticin] and $25 \mu \mathrm{l}$ were added to the Sil-15 cells. Sil-15 cells were grown in 96-well micro test plates (Becton-Dickinson) coated with $0.1 \%$ gelatin and each sample was tested on 6 separate wells. Control wells received either $25 \mu$ l of tissue culture medium or no addition. After overnight culture at $37^{\circ} \mathrm{C}$ in $5 \% \mathrm{CO}_{2}$, the medium was aspirated and the cells gently washed twice with chilled PBS. AtRA standards, used to determine relative amounts of RA synthesized in CNS tissue, were prepared as a $10^{-2} \mathrm{M}$ stock in DMSO and subsequently diluted in tissue culture medium to a final concentration of $10^{-6} \mathrm{M}, 10^{-7}$ $\mathrm{M}, 10^{-8} \mathrm{M}$ and $10^{-9} \mathrm{M}$. Solvent controls included equivalent concentrations of DMSO in tissue culture medium. Cells were then fixed in glutaraldehyde solution ( $1 \%$ glutaraldehyde in PBS, $0.1 \mathrm{mM} \mathrm{MgCl}_{2}$ ) on ice for $15 \mathrm{~min}$ followed by four additional PBS washes. Cells were developed for Lac $Z$ expression with $\mathrm{X}$-gal (5-bromo-4-chloro-3-indolyl- $\beta$-Dgalactopyranoside) as the substrate.

The percentage of $\beta$-galactosidase ( $\beta$-gal)-positive (blue) cells was determined by direct cell counts at $10 \times$ magnification for each well in the 96 well microtest plates. A one-way ANOVA was run on the cell counts of the RALDH assay to determine significance. Results are expressed as mean \pm SEM and deemed significant when $p<0.05$.

Tissue explant studies. Sil-15 reporter cells were seeded in $35 \mathrm{~mm}$ culture dishes and grown until $80-90 \%$ confluent $>48 \mathrm{~h}$. Acutely isolated snail brains were dissected under minimal light conditions, washed briefly in culture medium and explanted ( 1 per well, $n=3$ ) onto monolayers of Sil- 15 cells. Care was taken to ensure that explants adhered to the cell monolayers. After $18-20 \mathrm{~h}$, both explants and cells were washed with PBS, fixed and assayed for reporter gene expression as outlined above. An $18-20 \mathrm{~h}$ assay time was used to permit maximal induction of Lac Z expression in Sil-15 cells under conditions in which the snail brain explants remained viable. Cells were counted in an approximate $24 \mathrm{~mm}^{2}$ area immediately surrounding the CNS explant.

Retinoid extraction procedure. The extraction procedure used was a slightly modified version from that of Thaller and Eichele (1987). Sample preparations were obtained in the dark under yellow light. Lymnaea brain and hemolymph were isolated, immediately frozen in liquid nitrogen and stored at $-80^{\circ} \mathrm{C}$. Two replicates of $\sim 75$ brains and two replicates of $1.25 \mathrm{ml}$ of pooled hemolymph (from at least 5 different animals) were used. The average wet weight of pooled CNS samples was $227 \pm 9 \mathrm{mg}$. Samples were next thawed and homogenized in $500 \mu \mathrm{l}$ of ice-cold stabilizer buffer $(0.5 \%$ ascorbic acid, $0.5 \%$ EDTA in PBS at pH 7.3$)$. Fifty microliters from each homogenate were removed for protein estimation (Coomassie Blue dye binding assay, Bio-Rad). The remaining tissue suspension was then extracted with $500 \mu$ l of extraction solvent (8:1 ethyl acetate:methyl acetate, $0.5 \%$ butylated hydroxytoluene). Samples were then shaken vigorously for $30 \mathrm{~min}$ in a mechanical shaker (while on ice). The samples were centrifuged for $5 \mathrm{~min}$ and the upper organic phase was collected. Another $500 \mu \mathrm{l}$ of extraction solvent was added to the remaining aqueous phase and the procedure was repeated twice, each time pooling the supernatants. The total sample was then dried down in the dark under a light stream of nitrogen. Samples were then resuspended in $40 \mu \mathrm{l}$ of acetonitrile, centrifuged and transferred into amber HPLC sample vials.

High-pressure liquid chromatography. Chromatographic separation was performed on a Waters Spherisorb S5ODS2 C-18 column (particle size: $5 \mu \mathrm{m}$, column size: $4.6 \times 100 \mathrm{~mm}$ ). Injection volumes were $5 \mu \mathrm{l}$. Elution involved an initial mobile phase of $70 \%$ acetonitrile $(0.1 \%$ formic acid): $30 \% \mathrm{H}_{2} \mathrm{O}(0.1 \%$ formic acid $)$ changing via a linear gradient to $100 \%$ acetonitrile over a 30 min period at a flow rate of $0.5 \mathrm{ml} / \mathrm{min}$. Retention times were compared with commercial 9-cis-RA and atRA each dissolved in acetonitrile, and run individually or as a mix, separately from the tissue/hemolymph samples. Detection was set to $350 \mathrm{~nm}$ (Barua and Furr, 1998).

Mass spectrometry. Mass analysis was performed on a Bruker Model high capacity trap + liquid chromatography (LC)/mass spectrometry (MS)/MS system using electrospray in negative ion mode, connected online to an Agilent 1100 LC system with the conditions described above. The retention times for analysis of 9-cis-RA and atRA were confirmed, and the mass spectrometer was set up to detect $255 \mathrm{~m} / \mathrm{z}$, which is an intense fragment observed in MS2 when the parent ion $(299 \mathrm{~m} / \mathrm{z})$ is fragmented according to the following scheme: molecular anion $\left(299^{-}\right) \rightarrow\left(255^{-}\right)+\mathrm{CO}_{2}$.

This fragmentation behavior is common to both 9-cis-RA and atRA. The product anion at $255 \mathrm{~m} / \mathrm{z}$ was monitored and ion chromatogram peak areas for this species were used in the subsequent calibration. A calibration curve for both 9-cis-RA and atRA was created by analyzing four concentrations (between $50 \mathrm{pg} / \mu \mathrm{l}$ to $5 \mathrm{ng} / \mu \mathrm{l}$ ) of each standard, and plotting the area of the $255 \mathrm{~m} / \mathrm{z}$ peak (determined by QuantAnalysis) to its corresponding concentration. Analyses were performed in duplicate. Our HPLC/MS limit of detection $(0.5 \mathrm{pg} / \mu \mathrm{l})$ was set to a signal-to-noise ratio of 2:1, similar to that of McCaffery et al. (2002), and common for HPLC procedures.

Cell culture procedures. Lymnaea stagnalis were laboratory bred, kept in aerated, artificial pond water and fed lettuce. All cell culture procedures were performed as described previously (Syed et al., 1990; Dmetrichuk et al., 2006). Briefly, the central ring ganglia were isolated under sterile conditions, and after a number of antibiotic washes and subsequent enzymatic treatment, pinned down in a dissection dish and bathed in high osmolarity (L-15 derived) defined medium (DM) (Ridgway et al., 1991). The connective tissue sheath surrounding the ganglia was removed using a pair of fine forceps, and identified Visceral F (VF) somata were individually extracted by applying gentle suction via a fire-polished pipette. The somata were plated directly on poly-L-lysine-coated dishes (onto a glass coverslip) containing DM or (where stated) brain-conditioned medium (CM) (Wong et al., 1981). In DM alone, adult Lymnaea neurons do not normally extend neurites (Spencer et al., 1996), but adult neurons do regenerate their processes if cultured in CM (Syed et al., 1990; Spencer et al., 2000). CM contains many (as yet unidentified) neurotrophic factors and any 9-cis-or atRA that may be present in CM, falls below our detection limits with HPLC analysis. Either atRA or 9-cis-RA (Sigma-Aldrich) was dissolved in absolute ethanol (EtOH) and added to the culture dishes to give a final bath concentration of $10^{-7} \mathrm{M}$. Control dishes contained 
equivalent concentrations of $\mathrm{EtOH}$ in $\mathrm{DM}(0.001 \% \mathrm{EtOH})$. All dishes were placed in the dark immediately following addition of RA or EtOH, and were maintained at $22^{\circ} \mathrm{C}$. Experiments to investigate the role of calcium in the RA-induced outgrowth were conducted in DM that contained $50 \%$ of the normal levels of calcium ( $2 \mathrm{~mm}$ compared with $4 \mathrm{~mm}$ ). The $\mathrm{Ca}^{2+}$ channel blocker, cadmium, was added to some dishes to a final bath concentration of $10 \mu \mathrm{M}$.

Analysis of neurite outgrowth. To investigate the effects of atRA and 9-cis-RA on the induction of neurite outgrowth, cells were plated in DM, in the absence of any other neurotrophic factors. Neurite outgrowth from cells incubated in RA or EtOH control vehicle, was scored 2, 3 and $4 \mathrm{~d}$ after cell plating. Cells were viewed with an inverted microscope (Zeiss Axiovert 200) and imaging software (Northern Eclipse 7.0) was used to monitor and analyze the extent of neuronal outgrowth. To be included in the analysis, neurons must have adhered to the culture substrate (in the absence of adhesion, no outgrowth would occur). The number and total length of primary neurites emerging from each neuron was monitored at each time point. A two-way repeated measures (RM) ANOVA and Dunn's (Bonferroni corrected) post hoc test were used to determine statistical significance in the number and length of neurites in atRA and 9-cis-RA versus EtOH control conditions. Results are expressed as mean \pm SEM and deemed significant when $p<0.05$. In the separate series of experiments conducted to examine the combined effects of the 9-cis-and atRA isomers, as well as the effects of low $\mathrm{Ca}^{2+}$ and cadmium, one-way ANOVA and Dunn's (Bonferroni corrected) post hoc tests were run on the number and length of neurites produced on day 4. Chisquared tests were used to compare the number of cells that produced outgrowth in each condition and differences were considered significant when $p<0.05$. The occurrence of outgrowth was scored as the presence or absence of any neurite extension from the cell body (minimum $10 \mu \mathrm{M}$ in length) and the length of primary neurites was measured as the length on emergence from the cell body to the very tip of the neurite.

Electrophysiology. For intracellular recordings, conventional electrophysiological techniques were used as described previously (Spencer et al., 2000, Dmetrichuk et al., 2006). Glass microelectrodes (resistance $20-40 \mathrm{M} \Omega$ ) containing a saturated solution of $\mathrm{K}_{2} \mathrm{SO}_{4}$, were used to impale the neurons under RA and EtOH culture conditions. The electrophysiological signals were amplified (IR-283, Cygnus Technology) and displayed using a data acquisition system designed by T. MacDonald (Electronics Department, Brock University, St. Catharines, ON, Canada). Electrophysiological recordings were performed blindly, in that the culture condition was unknown at the time of the recordings. Recordings were only made from cells that were adhered to the substrate and were morphologically "intact" (i.e., had not ruptured). In all recordings, cells were assessed for their ability to fire spontaneous or induced action potentials (0.1-0.5 pA depolarizing current injection). A one-way RM ANOVA and Dunn's (Bonferroni corrected) post hoc analysis was performed to compare the resting membrane potentials (RMPs) of the cells between groups. Results were expressed as mean \pm SEM and differences were deemed significant if $p<0.05$. Chi-squared tests were performed to compare the number of cells that fired action potentials (APs) following incubation in RA versus EtOH control. Results were expressed as the percent of cells fired, and differences were deemed significant if $p<0.05$. Analysis of AP shape was performed with Chart software (v6.0, AD Instruments). A minimum of three action potentials were analyzed for each cell and the amplitude in millivolts (from $\mathrm{V}_{\text {rest }}$ to the peak of the action potential), the half-amplitude duration (in milliseconds), and the fall time in milliseconds (10-90\% of the falling phase of the action potential) were measured, and values expressed as mean \pm SEM. Values for 9-cisand atRA-incubated cells were compared using Student's $t$ test.

Growth cone turning assay. For growth cone turning assays, individual VF neurons were cultured in CM and allowed to extend neurites for a minimum of $18 \mathrm{~h}$. Only actively growing neurites were used. Growth cones were monitored for at least $1 \mathrm{~h}$ before exposure to a local gradient of 9-cis-RA. A pipette containing 9-cis-RA ( $10^{-5}$ M; Sigma-Aldrich) was placed in close proximity to the growth cone and 9-cis-RA was pressure ejected using an Eppendorf-Femtojet ( $10 \mathrm{~min}$ continuous pulses at 6-15 $\mathrm{hPa}$, with $5 \mathrm{~min}$ between, for $0.5-3 \mathrm{~h}$; pipette tip diameter: $3-7 \mu \mathrm{m}$ ). Digital images were captured, and growth cone turning was analyzed using Northern Eclipse Software. The maximum turning angles were measured and expressed as mean \pm SEM.

\section{Results}

We have previously shown that the neurotrophic and chemotropic effects of atRA observed in vertebrate neurons, are conserved in neurons of an invertebrate species (Dmetrichuk et al., 2006). However, despite some evidence for physiological effects of exogenously applied RA in invertebrates, there is less evidence for either the presence or synthesis of endogenous RA in an invertebrate species, compared with vertebrates. The first aim of this study was thus to investigate, using a reporter cell line, whether RA was synthesized or released in the CNS of the mollusc Lymnaea stagnalis.

\section{Endogenous RALDH activity in Lymnaea CNS and hemolymph}

Once dietary Vitamin A (or retinol) is taken up by a cell, it is enzymatically converted to retinal. Retinal is then converted to RA by RALDHs (Maden and Hind, 2003). Many studies that measure endogenous retinoids thus commonly explore RALDH activity. Here, RALDH activity was determined in tissue samples and the reaction products then added to a retinoid-sensitive reporter cell line with the ability to detect nM quantities of RA (Wagner, 1998).

Figure $1 A$ shows $\beta$-gal expression in fixed, control Sil- 15 cells. The average percentage of $\beta$-gal positive cells determined from 12 replicate wells was $1.2 \pm 0.15 \%$. The low but reproducible number of positive cells in the absence of exogenous retinoids could be due to residual retinoids in the calf serum. Reaction products of RALDH assays from Lymnaea CNS extracts $(n=12)$ produced an average of $4.6 \pm 0.46 \% \beta$-gal-positive cells (Fig. $1 B$ ). A oneway ANOVA analysis revealed a significant difference between the control and CNS conditions $(p<0.0001)$, suggesting the presence of endogenous RALDH activity and the synthesis of RA in the adult Lymnaea CNS.

\section{Retinoid release from the adult Lymnaea CNS}

Perhaps a more relevant measure of endogenous retinoid activity, particularly when studying the neurotrophic role of RA in regeneration, is to measure its release directly from isolated adult CNS tissue. Figure $1 C$ demonstrates $\beta$-gal activity in fixed Sil- 15 cells following addition of a CNS explant. The highest percentage of active cells was found closest to the CNS explant, and this number declined at increasing distances from the explant (data not shown), suggesting the release and diffusion of RA from the brain tissue. We estimated the concentration of released RA to be $\sim 10^{-6} \mathrm{M}$ closest to the brain explants, and $\sim 10^{-10} \mathrm{M}$ at the farthest point away. This value was calculated from a standard curve generated with the addition of synthetic atRA $\left(10^{-6} \mathrm{M}\right.$ shown in Fig. 1D).

\section{HPLC/MS of atRA and 9-cis-RA standards}

The above data strongly suggest the presence of an active RA metabolic pathway in the adult Lymnaea CNS. However, the assay used did not allow us to determine which isomer was present and synthesized in the CNS. Thus, we next used HPLC and MS to determine whether atRA and/or 9-cis-RA were present in the CNS.

To determine whether atRA and 9-cis-RA isomers could be separated and detected by HPLC, a mixture of the two synthetic compounds was first analyzed. Despite similarities in structure and polarity, each isomer could be separately identified in the 

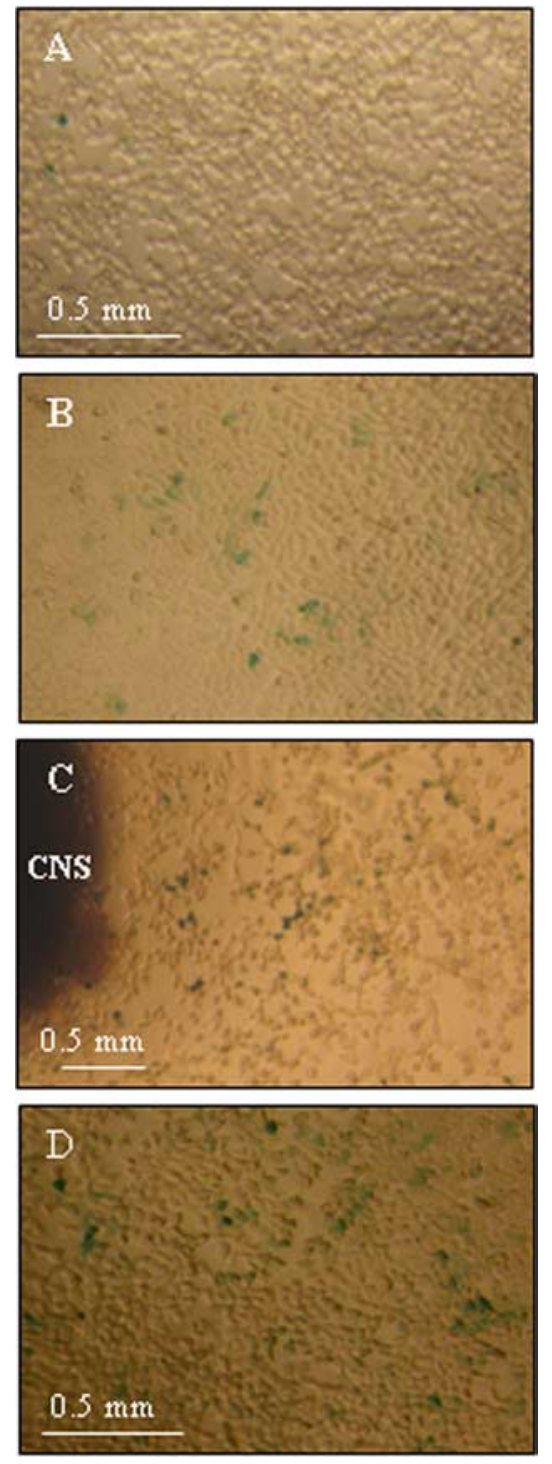

Figure 1. RALDH activity in brain extracts of Lymnaea stagnalis. A, Sil-15 reporter cells stained for $\beta$-gal activity after addition of culture medium. $\boldsymbol{B}$, A similar field of Sil-15 reporter cells stained for $\beta$-gal activity after the addition of $25 \mu$ l of RALDH reaction product with Lymnaea CNS extract as the substrate. Note a significant increase in the number of $\beta$-galpositive (blue) cells. C, RA release by Lymnaea CNS explants. Cells stained for $\beta$-gal activity as in $\boldsymbol{A}$ and $\boldsymbol{B}$ above. $\boldsymbol{D}$, Sil-15 reporter cells following the addition of $10^{-6} \mathrm{M}$ atRA.

same sample as illustrated in Figure 2. 9-cis-RA eluted at $14.6 \mathrm{~min}$ and atRA at $15.1 \mathrm{~min}$, with minimal overlap of the two peaks. Both standards were also run separately (data not shown), with no effect on relative retention times. These standard samples were further analyzed after HPLC separation by MS. The atRA standard molecular weight was measured to be $299.1 \mathrm{~m} / \mathrm{z}$ (Fig. 3A). Once fragmented, the molecular weight was measured to be 255.1 $\mathrm{m} / \mathrm{z}$ (Fig. $3 \mathrm{~B}$ ) corresponding to the loss of $\mathrm{CO}_{2}$. Analysis of 9-cis-RA yielded similar results (Fig. $3 C, D$ ) as expected, since both compounds are isomers with the same molecular weight and functional group.

\section{HPLC/MS analysis of adult Lymnaea stagnalis CNS and hemolymph extracts}

Our next objective was to determine the relative abundance of each isomer in the CNS of adult Lymnaea. Due to a previous report by Munno et al. (2000) which suggested that some trophic

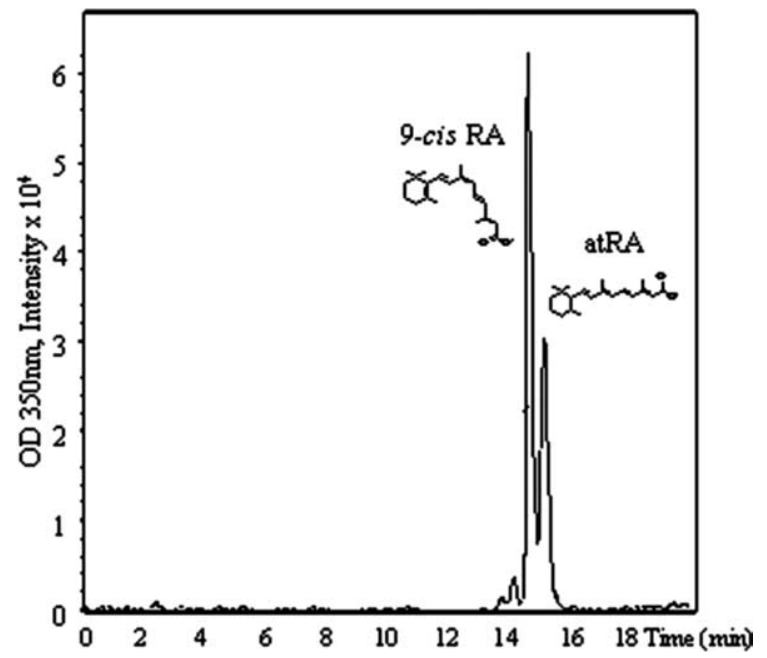

Figure 2. HPLC separation of 9-cis-RA and atRA standards run as a mixture. 9-cis-RA elutes at $14.6 \mathrm{~min}$ and atRA elutes at $15.1 \mathrm{~min}$.

A
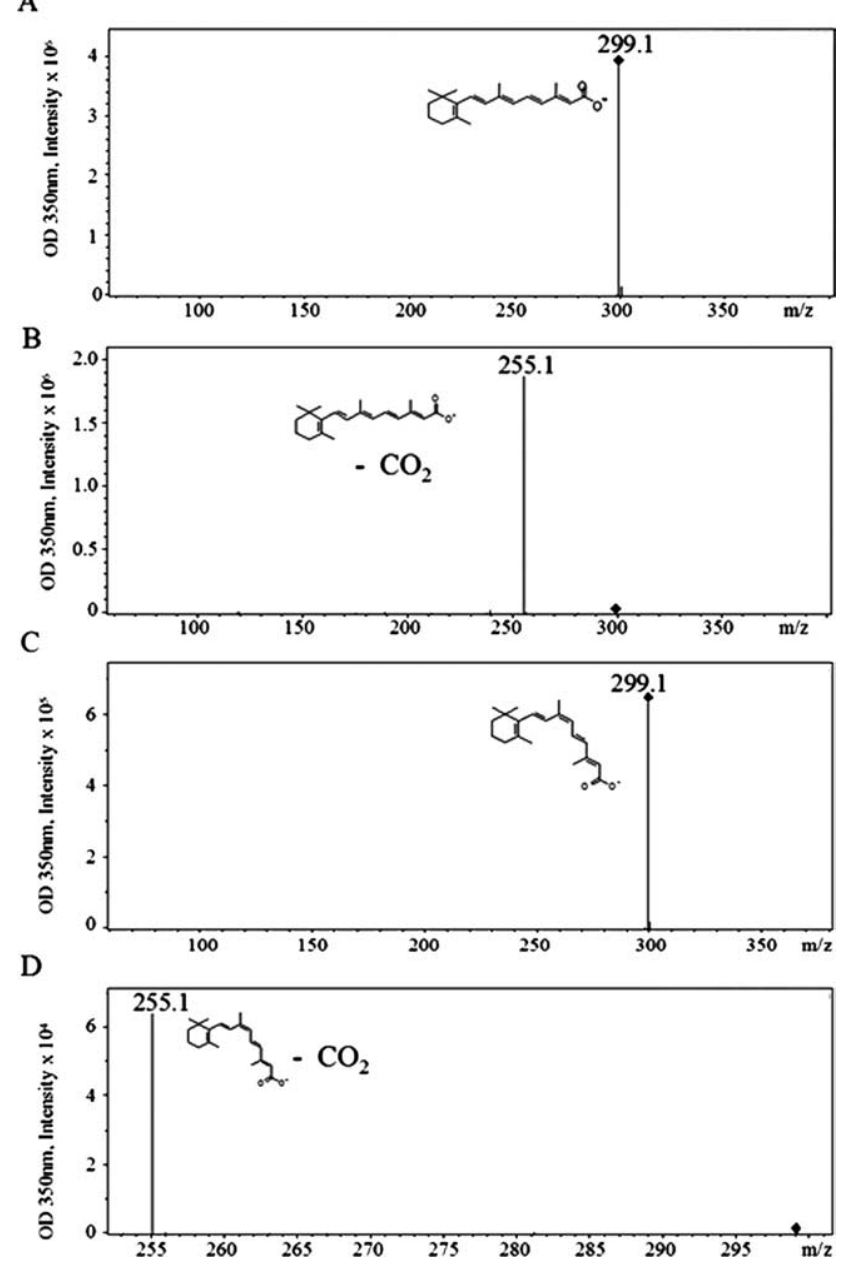

Figure 3. Mass spectra and electrospray ionization of atRA and 9-cis-RA standards. A, AtRA standard molecular weight is determined to be $299.1 \mathrm{~m} / z$. B. Electrospray ionization of atRA. C, 9-cis-RA standard molecular weight is determined to be $299.1 \mathrm{~m} / z$. D, Electrospray ionization of 9-cis-RA.

factors are carried in the invertebrate hemolymph, extractions were performed on the hemolymph as well as the CNS.

HPLC was performed on samples from two independent pools of isolated adult Lymnaea CNSs. Two peaks were deter- 
mined to be of interest (Fig. $4 A$ ) as they were within 0.1 retention time of RA standards. Peak 1 had a retention time of 14.6 min, corresponding to the retention time for the 9-cis-RA standard, while peak 2 eluted at $15.1 \mathrm{~min}$, characteristic of atRA.

Both samples were further investigated by MS. The mass for each peak was determined to be $299 \mathrm{~m} / \mathrm{z}$. This again corresponds to the molecular weights of both 9-cis-RA and atRA. After ionization, the mass for each resulting fragment was determined to be $255 \mathrm{~m} / \mathrm{z}$, confirming the loss of a carboxylic acid group. These data were consistent in duplicate experiments and strongly indicated the presence of both the 9-cis-RA and atRA in the adult Lymnaea CNS.

HPLC was also performed on two independent pools of isolated hemolymph (Fig. $4 B$ ). Once again, two peaks were found within 0.1 retention time for each RA standard. Peak 1 had a retention time of $14.6 \mathrm{~min}$, which corresponded to the 9-cis-RA standard, and peak 2 had a retention time of 15.0 min that closely corresponded to that of atRA.

To confirm their identities as all-trans and 9-cis-RA, the peaks were further analyzed by MS. The molecular mass for each peak was determined to be $299 \mathrm{~m} / \mathrm{z}$. Again, this corresponded to the molecular weights of both 9-cis-RA and atRA. Additionally, the mass for each fragmented peak was determined to be $255 \mathrm{~m} / \mathrm{z}$. These data were consistent between duplicate experiments and strongly indicated the presence of both 9-cis-RA and atRA in the adult Lymnaea hemolymph.

\section{Estimation of endogenous retinoid levels}

A calibration curve was generated for both 9-cis-RA and atRA by analyzing four concentrations (between $50 \mathrm{pg} / \mu \mathrm{l}$ to $5 \mathrm{ng} / \mu \mathrm{l}$ ) of each standard, and plotting the area of the $255 \mathrm{~m} / \mathrm{z}$ peak (determined by QuantAnalysis) to its corresponding concentration. From this analysis, with an estimated RA extraction efficiency of 70\% (Scadding and Maden, 1994), we calculated endogenous atRA concentration in the CNS tissue to be $77.14 \mathrm{pg} / \mathrm{mg}$ wet weight $(693 \mathrm{nM})$, while in the hemolymph, the concentration was estimated at $17.22 \mathrm{pg} / \mathrm{mg}$ wet weight (155 nM) (Fig. 5). For 9-cis$\mathrm{RA}$, the values were slightly lower; $42.20 \mathrm{pg} / \mathrm{mg}$ wet weight (380 $\mathrm{nM}$ ) for CNS tissue and $13.30 \mathrm{pg} / \mathrm{mg}$ wet weight $(120 \mathrm{~nm})$ for hemolymph (Fig. 5).

9-cis-RA induced comparable neurite outgrowth as atRA from cultured neurons

Previously, we have shown that atRA induced neurite outgrowth from adult Lymnaea neurons in cell culture (Dmetrichuk et al., 2006). However, it is not yet known whether 9-cis-RA exerts neurotrophic effects in the nervous system. After determining from HPLC and MS that both atRA and 9-cis-RA are present in the CNS, we next set out to determine whether 9-cis-RA has neuriteinducing effects and if so, to compare the effects of 9-cis-RA with atRA. To avoid potential complications of comparing data across different culture days, atRA experiments were repeated in this study and run at the same time as the 9-cis-RA experiments. This allowed us to directly compare the effects of the two isomers under identical culture conditions. In this series of experiments, only EtOH was used as a control, as we have previously shown no

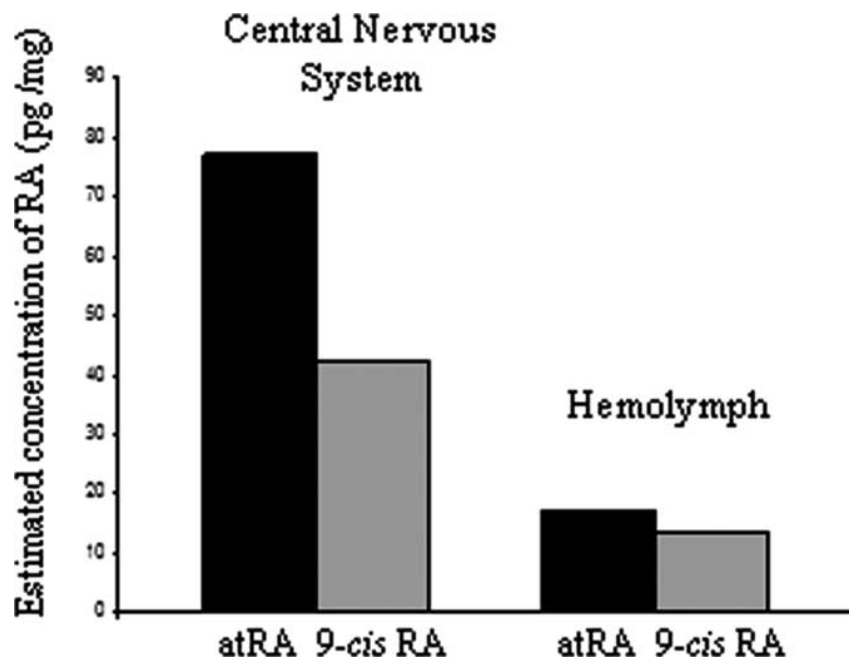

Figure 5. Estimated relative concentrations of 9-cis-RA and atRA in the adult Lymnaea CNS and hemolymph.

difference between $\mathrm{EtOH}$ and DM alone, on outgrowth from VF neurons (Dmetrichuk et al., 2006).

We found that in the presence of either atRA or 9-cis-RA $\left(10^{-7} \mathrm{M}\right)$, significantly more cells produced neurite outgrowth after $2 \mathrm{~d}$ in culture than in the $\mathrm{EtOH}$ control conditions. Figure $6 \mathrm{~A}$-iii shows representative examples of cells with neuritic extension in 9-cis-RA, atRA and EtOH. Cells cultured in the EtOH vehicle solution were significantly less likely to produce neurite outgrowth. A $\chi$-square test revealed no significant difference in the number of cells with outgrowth between 9-cis-RA and atRA $\left(\chi^{2}=2.57, \mathrm{df}=1 ; p>0.05\right.$, n.s. $)$, although both 9-cis-RA $(p<$ $0.01)$ and atRA $(p<0.001)$ induced significantly more cells to produce outgrowth than the EtOH control condition (Fig. $6 \mathrm{~B}$ ).

Next, we investigated the extent of neurite regeneration in the different conditions. Cells that were plated in 9-cis-RA $(n=42)$, atRA $(n=32)$ or control $(\mathrm{EtOH})(n=47)$ were assessed for neurite outgrowth after 2, 3 and $4 \mathrm{~d}$ in culture. The total length of primary neurites in cells cultured with 9-cis-RA and atRA was not significantly different from each other after $4 \mathrm{~d}$ in culture $(p>$ 0.05 , n.s.) (Fig. 6C), although both were significantly greater compared with control $(p<0.01)$. Additionally, we found that 
A

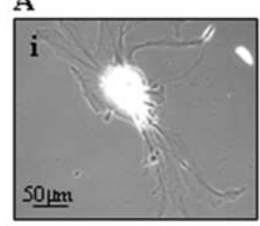

9-cis RA

27 of 42 grew

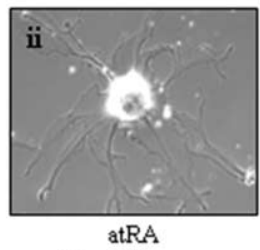

26 of 32 grew

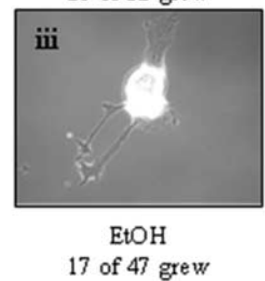

B

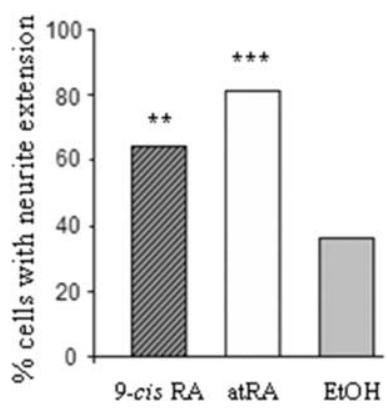

Figure 6. 9-cis-RA and atRA significantly increased neurite outgrowth. $\boldsymbol{A}$, Representative examples of identified VF cells showing neurite outgrowth after $2 \mathrm{~d}$ in culture (DM) after treatment with 9-cis-RA (i), atRA (ii), and EtOH (iii) vehicle solution (example of a cell with neurite outgrowth). $\boldsymbol{B}$, Histogram showing that the percentage of cells producing neurite outgrowth in 9-cis-RA and atRA on day $2\left(10^{-7} \mathrm{M}\right)$ was significantly greater than the percentage of cells producing outgrowth in vehicle control (EtOH). There was no significant difference between 9-cis-RA and atRA. $\left({ }^{* *} p<0.001,{ }^{* *} p<0.01\right.$; compared with EtOH controls). C, The total length of primary neurites extended was significantly greater on days 2, 3 and 4 in both 9-cis-RA and atRA compared with EtOH controls. D, Also, the number of primary neurites extended was significantly greater on days 2,3 and 4 in 9-cis-and atRA compared with controls. ${ }^{* *} p<0.01$ (statistical comparison shown only for day 4 and compared with EtOH control conditions).

cells in both 9-cis-RA and atRA conditions showed significantly more primary neurites after $4 \mathrm{~d}$ in culture, compared with the cells in $\mathrm{EtOH}(p<0.01)$. However, there was no significant difference between the number of primary neurites extended after $4 \mathrm{~d}$ between 9-cis-RA and atRA ( $p>0.05$, n.s.) (Fig. 6D).

We next aimed to determine whether culturing cells in both 9-cis-RA and atRA in the same dish would produce significantly different levels of outgrowth than either isomer alone. This may indicate whether the two isomers have additive or competitive effects on neurite outgrowth. A separate series of experiments were conducted in which cells were either plated in 9-cis-RA $\left(10^{-7} \mathrm{M}\right)$, atRA $\left(10^{-7} \mathrm{M}\right)$ or a combination of both 9-cis-RA $\left(10^{-7} \mathrm{M}\right)$ and atRA $\left(10^{-7} \mathrm{M}\right)$. It was found that there was no significant difference in the percentage of cells that had initiated outgrowth between any of the conditions on day 4 (9-cis-RA alone: $54.8 \%$ (17/31); atRA alone: $69.2 \%$ (18/26); 9-cis-RA and atRA together: $73.1 \%(n=19 / 26) ; p>0.05)$, suggesting that culturing in both isomers did not have an additive effect. Furthermore, there was no significant difference in either the number of neurites $(p>0.05)$ or the length of neurites $(p>0.05)$ on day 4 , between cells plated in one isomer alone compared with both 9-cis-RA and atRA isomers together.

The cellular and molecular mechanisms of RA-induced outgrowth have not been extensively studied, although it is well known that optimal intracellular calcium $\left(\mathrm{Ca}^{2+}\right)$ levels are required for neurite outgrowth and extension (Kater and Mills, 1991). In the next series of experiments, we lowered extracellular $\mathrm{Ca}^{2+}$ concentrations to $50 \%$ of normal DM, and used the $\mathrm{Ca}^{2+}$ channel blocker, cadmium (10 $\mu \mathrm{M}$, Feng et al., 2000), to investigate a potential role of $\mathrm{Ca}^{2+}$ in the 9-cis-RA-mediated neurite outgrowth. The mere lowering of extracellular $\mathrm{Ca}^{2+}$ and the presence of cadmium, should not adversely affect neurite outgrowth as these culturing conditions have previously been shown to support robust outgrowth from Lymnaea neurons in conditioned media (Feng et al., 2000). However, if $\mathrm{Ca}^{2+}$ influx plays an important role in the RA-induced outgrowth, we would predict that significant reductions in outgrowth would be observed. Cells were plated in one of three conditions: (1) low $\mathrm{Ca}^{2+}$ and 9-cis-RA, (2) low $\mathrm{Ca}^{2+}$, cadmium and 9-cis-RA, or (3) normal DM and 9-cis-RA, and the percentage of cells grown, number of primary neurites, and length of neurites on day 4 were recorded and analyzed. We found no significant difference in the percentage of cells grown (low $\mathrm{Ca}^{2+}$ and 9-cis-RA: 63.3\% $(n=19$ ) 30); low $\mathrm{Ca}^{2+}$, cadmium and 9-cis-RA: $72.1 \%(n=31 / 43)$; normal DM and 9-cisRA: $75.9 \%(n=22 / 29)$ or the number of neurites (low $\mathrm{Ca}^{2+}$ and 9-cis-RA: $4.8 \pm$ 0.5 ; low $\mathrm{Ca}^{2+}$, cadmium and 9-cis-RA: $4.7 \pm 0.5$; normal DM and 9-cis-RA: $5.2 \pm$ $0.8 ; p>0.05)$ across all three conditions. However, there was a significant reduction $(p<0.01)$ in the length of neurites in low $\mathrm{Ca}^{2+}$ and cadmium $(293.1 \pm 36.6 \mu \mathrm{m})$ compared with the other two conditions (low $\mathrm{Ca}^{2+}$ and 9-cis-RA: $512.4 \pm 80.9 \mu \mathrm{m}$; normal DM and 9-cis-RA: $735.6 \pm 150.5 \mu \mathrm{m}$ ). These data suggest that although $\mathrm{Ca}^{2+}$ influx may not play a significant role in the induction of outgrowth by 9 -cis-RA, it may play an important role in the RA-induced extension of outgrowth.

\section{Cells retained their electrical excitability in 9-cis-RA over time}

We have previously shown that in addition to inducing neurite outgrowth, atRA also exerted neurotrophic actions by extending the electrical viability of VF neurons in culture (Dmetrichuk et al., 2006). Therefore, we next investigated the ability of 9-cis-RA to maintain RMP and electrical excitability of neurons in culture. Cells were tested after $4 \mathrm{~d}$ in culture following incubation in 
9-cis-RA, atRA or EtOH culture conditions (as this was the time point previously shown to produce optimal differences for atRA; Dmetrichuk et al., 2006). As shown in Figure 7A, the RMP of cells in 9-cis-RA $(-52.38 \mathrm{mV} \pm 2.51 \mathrm{mV})$ and atRA $(-47.85 \mathrm{mV} \pm 4.54 \mathrm{mV})$ were significantly more negative than those of cells in EtOH control conditions $(-35.71 \mathrm{mV} \pm$ $3.60 \mathrm{mV})(p<0.01, p<0.05$ respectively). However, we found that the RMP of cells cultured in 9-cis-RA and atRA were not significantly different from each other ( $p>0.05$, n.s.). The percentage of cells that were electrically excitable (spontaneous or induced firing) on day 4 of culture were significantly greater in 9-cis-RA (11 of $13,85 \%$ ) and atRA ( 14 of $20,70 \%$ ) versus those cultured in the control EtOH solution ( 4 of $17,24 \%$; $p<0.01$ ). Similarly, the effects of 9-cis-RA and atRA on firing were not significantly different from each other $(p>0.05$, n.s) (Fig. $7 B)$. Figure $7 C$ shows representative intracellular electrophysiological recordings from cells cultured in 9-cis-RA (i), atRA (ii) and EtOH (iii) respectively. The traces show that following depolarizing current injection, the cells cultured in 9-cis-RA or atRA fired APs on day 4 , whereas the cell cultured in vehicle $(\mathrm{EtOH})$ control failed to fire in response to current injection on day 4. APs obtained on day 4 in both 9-cis-and atRA were analyzed for spike amplitude (9-cisRA: $55.1 \pm 2.1 \mathrm{mV}$; atRA: $53.9 \pm 4.9 \mathrm{mV}$ ), half-amplitude spike duration (9-cis-RA: $17.3 \pm 3.7 \mathrm{~ms}$; atRA: $27.2 \pm 4.2 \mathrm{~ms})$, and fall time (9-cis-RA: $27.0 \pm 4.3 \mathrm{~ms}$; atRA: $28.4 \pm 3.8 \mathrm{~ms}$ ) to investigate whether there were any significant differences in spike shape between the 9-cis-RA and atRA isomers (EtOH conditions could not be compared because of the low number of cells firing on day 4$)$. We found no significant differences $(p>0.05)$ in any of the recorded values between 9 -cis-and atRA.

\section{9-cis-RA induced positive growth cone turning responses in vitro}

The data shown above demonstrate that both atRA and 9-cis-RA exert similar neurotrophic effects on VF neurons, to both induce neurite outgrowth and maintain electrical viability for longer periods in cell culture. We next aimed to determine whether the 9-cis-RA isomer could exert comparable chemotropic effects to those we have previously observed with atRA (Dmetrichuk et al., 2006). As it was not imperative to perform these particular experiments in parallel with atRA, only 9-cis-RA was exogenously applied to growth cones in this study.

VF neurons were cultured in CM and following outgrowth (1-2 d), were tested for growth cone turning in response to an exogenous source of focally applied 9-cis-RA. Growth cones were first monitored for at least $1 \mathrm{~h}$ to ensure that no spontaneous changes in direction occurred. As previously shown with atRA, 9-cis-RA $\left(10^{-5} \mathrm{M}\right.$ in pipette) also produced positive growth cone turning (Fig. $8 A$ ). Figure $8 B$ shows a histogram depicting the maximum turning angle of growth cones toward 9-cis-RA, with each bar on the histogram representing one growth cone from one cell. We found that the mean maximum turning angle toward 9-cis-RA $\left(60.38^{\circ} \pm 8.14^{\circ} ; n=8\right)$ was not significantly different from that previously shown with atRA $\left(66.1^{\circ}\right)$ (Dmetrichuk et al., 2006) but was significantly greater than EtOH or DM controls $(p<0.01)$. Thus both atRA and 9-cis-RA isomers showed similar effects to induce positive growth cone turning in vitro. This is the first study to demonstrate chemotropic effects of 9-cis-RA on neurons from any species.

\section{Discussion}

In this study, we have provided the first direct evidence for the existence of both atRA and 9-cis-RA isomers in the CNS of an invertebrate species and provide important evidence for a potential physiological role for 9-cis-RA in neuronal regeneration. Moreover, this is the first study in any species to show that 9-cis-RA exerts similar chemotropic effects as atRA to produce growth cone turning in cultured neurons.

Our data using the Sil-15 reporter cell line first indicated that the molluscan CNS, similar to its vertebrate counterpart, is capable of synthesizing RA from retinal through the actions of endogenous RALDH. In support of this conclusion, Lymnaea sequences encoding a full-length retinal dehydrogenase (C. J. Carter, G. E. Spencer, unpublished observations), and four reti- 

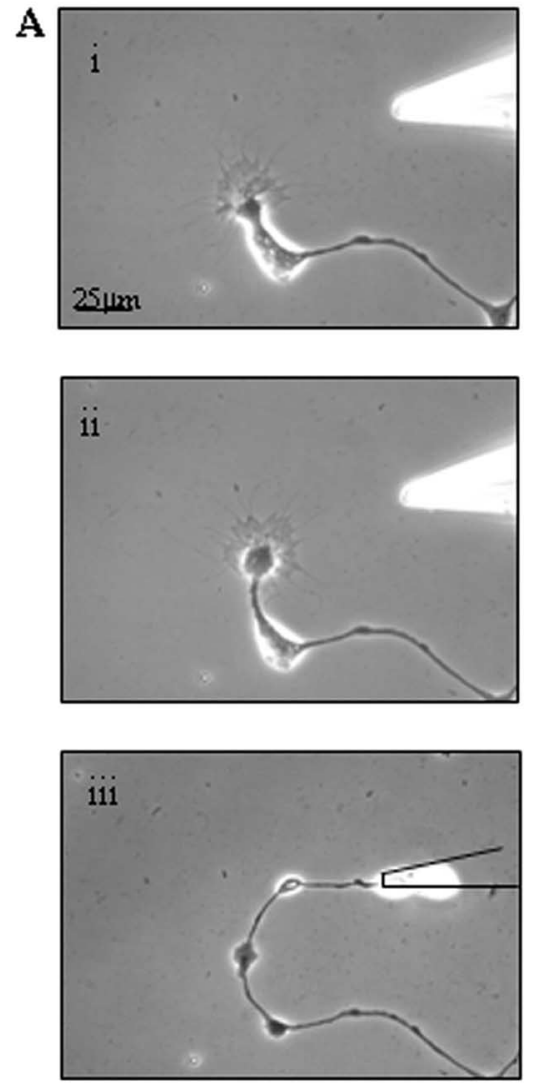

B

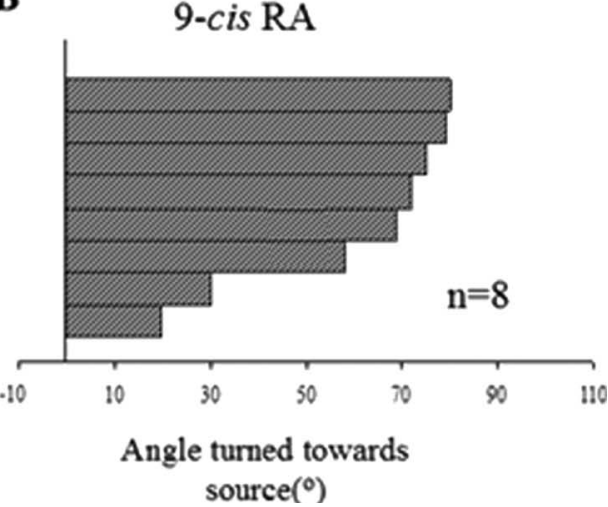

Figure 8. 9-cis-RA induced positive growth cone turning. $A, A$ VF cell was cultured in CM for $2 \mathrm{~d}$ to allow for neurite outgrowth and then monitored for $1 \mathrm{~h}$ to ensure no spontaneous changes in growth cone direction occurred. $\boldsymbol{i}$, Control image before the directional, pulsatile application of $10^{-5} \mathrm{M} 9$-cis-RA via a micropipette $(t=0 \mathrm{~min})$. ii, Eighteen minutes after the start of RA application, the growth cone turned toward the source of 9 -cis-RA. iii, Fourteen hours after the removal of the local source of 9-cis-RA, the growth cone continued to grow in the new direction. $B$, The histogram depicts the maximum turning angles of growth cones exposed to 9-cis-RA $\left(10^{-5} \mathrm{M}\right)$. Each bar on the histogram represents one growth cone from one cell.

nol dehydrogenases and a putative cellular retinoic acid binding protein have been detected, all of which show considerable homology to vertebrate sequences. These cell line experiments did not provide any specific information regarding which endogenous RA isomers were being synthesized and released and it was only through use of HPLC and MS that we identified both 9-cis-RA and atRA in the CNS of Lymnaea.

atRA has previously been detected in both embryonic and adult nervous systems from a number of vertebrate species (Horton and Maden, 1995; Maden et al., 1998b; Hoover et al., 2001,
Werner and DeLuca, 2002). However, 9-cis-RA detection in vertebrates is rare; in the mouse embryonic spinal cord, 9-cis-RA was detected only after atRA levels were artificially elevated (Ulven et al., 2001) and others report that 9-cis-RA levels are below their limits of detection (Schmidt et al., 2002). The relevance of a physiological role for 9-cis-RA has thus been questioned (Horton and Maden, 1995), although there is recent evidence in the vertebrate nervous system to suggest its role in reducing the production of inflammatory cytokines from activated glia (Xu and Drew, 2006).

In invertebrates, the presence of RA has been indirectly implicated by the presence of retinoic acid binding proteins in the insect (Mansfield et al., 1998) shrimp (Gu et al., 2002) and marine sponge (Biesalski et al., 1992). However, in contrast to vertebrates, where 9-cis-RA has been difficult to detect, both atRA and 9-cis-RA isomers have been directly detected in the prevertebrate amphioxus (Dalfó et al., 2002), fiddler crab limb blastemas (Hopkins, 2001) and recently, the locust embryo (Nowickyj et al., 2008). Although RA has been shown to alter eye formation in the mollusc Lymnaea (Créton et al., 1993) and vitamin A was detected in the mollusc Helix pomatia (Eakin and Brandenburger, 1968), little is known about endogenous retinoids in molluscs. Significantly, the direct detection of RA (either atRA or 9-cis-RA) in the CNS of any invertebrate species has not previously been shown. We report here that both atRA and 9-cis-RA are present in the molluscan CNS (and hemolymph) in nM concentrations, although the concentration of atRA was estimated to be slightly higher than 9-cis-RA. The estimated concentrations reported in this study are in accordance with previous detection levels of RA in vertebrate limb tissue (Thaller and Eichele, 1987; Scadding and Maden, 1994) as well as locust embryo (Nowickyj, 2008). Interestingly, in the locust embryo, 9-cis-RA was slightly more abundant than atRA (Nowickyj et al., 2008).

9-cis-RA has been difficult to detect previously, either due to its low endogenous concentration (Schmidt et al., 2002) or its potential for isomerization to atRA and/or 13-cis-RA (Kraft and Juchau, 1993; Shirley et al., 1996). We cannot rule out the possibility that the 9-cis-RA detected in our samples arose as a consequence of its isomerization from atRA during the extraction procedure. We feel this is unlikely though, as after a $3 \mathrm{~d}$ exposure of atRA to a 12 -h light/dark cycle at $23^{\circ} \mathrm{C}$, nuclear magnetic resonance (NMR) and UV analyses revealed only trace amounts of 9-cis-RA (our unpublished observations). Moreover, atRA is very stable in acetonitrile for up to $2 \mathrm{~d}$ at room temperature (Kane et al., 2005). Our samples were immediately frozen in liquid nitrogen and protected from heat, air and light, thus minimizing the potential effects of endogenous isomerases. In addition, neither Nowickyj et al. (2008), or Biesalski et al. (1992) detected any significant trans to cis-isomerization, while Brodeur et al. (2007) also failed to demonstrate endogenous RA isomerase activity in cultured HeLa cells. Indeed, the existence of cis-retinol isomers and the enzymes capable of oxidizing them to their characteristic RAs (Labrecque et al., 1995, Driessen et al., 1998, Romert et al., 1998, Paik et al., 2005), strongly suggests that 9-cis-RA (and other cis-RA isomers) may be synthesized independently from atRA.

Having detected endogenous 9-cis-RA in the CNS at relatively similar concentrations to atRA, we next demonstrated for the first time, that 9-cis-RA was capable of acting as a neurotrophic and chemotropic factor on cultured neurons. In the absence of any other trophic factors, 9-cis-RA induced significantly more neurite outgrowth compared with controls. Additionally, we showed that 9-cis-RA was capable of maintaining the electrical excitability and RMP of neurons after $4 \mathrm{~d}$ in culture. These neurotrophic effects occurred at $10^{-7} \mathrm{M}$ bath concentrations of RA, 
concentrations similar to the endogenous CNS concentrations estimated in the first part of our study, further supporting a physiological role for 9-cis-RA. Although we did not investigate whether there was a significant correlation between the trophic effects of 9-cis-RA on neurite induction and electrophysiological properties of the cells, we have previously shown that these trophic effects of atRA appeared to be independent of each other (Dmetrichuk et al., 2006). That is, we previously suggested that atRA was not inducing outgrowth from the Lymnaea neurons merely by enhancing survival of the cells, and it is likely that this is also true for 9-cis-RA.

Interestingly, these neurotrophic effects exerted by 9-cis-RA were not significantly different from the effects produced by atRA on the same cell type. The capability of the two isomers to act in a similar manner has been previously documented; when a murine septal cell line was treated with atRA or 9-cis-RA, both increased the expression of choline acetyltransferase mRNA threefold compared with controls (Pedersen et al., 1995). Plum and ClagettDame (1995) showed that both atRA and 9-cis-RA inhibited the proliferation of human neuroblastoma cells and induced RAR $\beta$ mRNA expression with equal capabilities. Other studies show however, that 9-cis-RA is more potent in its differentiating and anti-proliferative capabilities (Han et al., 1995; Lovat et al., 1997). Opposite effects of 9-cis-RA and atRA have also been observed (Hellemans et al., 1999), but these did not involve the nervous system.

A novel finding from this study was that 9-cis-RA induced positive growth cone turning and this is the first evidence from any species that 9-cis-RA may act as a potential guidance molecule in the nervous system. In vertebrates, Maden et al. (1998a) showed that embryonic chick neurons grew toward a source of atRA $\left(2 \times 10^{-6} \mathrm{M}\right)$ and we have previously shown that exogenous atRA induced directed outgrowth from the adult newt spinal cord (Dmetrichuk et al., 2005). Although directed outgrowth with atRA has been observed, our previous studies with Lymnaea cultured neurons were the first demonstration of individual growth cone turning in response to atRA (Dmetrichuk et al., 2006). We now demonstrate that 9-cis-RA can produce a very similar turning response in growth cones from the same identified neurons, providing the very first evidence for a chemotropic response by this specific isomer, and suggesting a physiological role in axonal pathfinding.

Very little is currently known about the cellular and molecular mechanisms underlying RA-induced neurite outgrowth. It is also not yet known whether 9-cis-RA and atRA's chemotropic and neurotrophic effects occur through an independent mechanism or by a shared mechanism. Our experiments showed that cells plated in both 9-cis-and atRA together, did not demonstrate significantly different levels of outgrowth, suggesting that the two isomers did not have an additive effect. However, it may be that our outgrowth assay is not sensitive enough to show additive effects as a result of merely doubling the RA concentration and previous unpublished experiments by our laboratory using different doses of atRA $\left(10^{-7} \mathrm{M}\right.$ and $\left.5 \times 10^{-6} \mathrm{M}\right)$ support this notion. As $\mathrm{Ca}^{2+}$ is well known to play a role in neurite outgrowth and extension (for review see Kater and Mills, 1991), we tested whether $\mathrm{Ca}^{2+}$ influx may play a role in the 9-cis-mediated outgrowth in the Lymnaea neurons. We found that in the presence of both low extracellular $\mathrm{Ca}^{2+}$ and the $\mathrm{Ca}^{2+}$ channel blocker, cadmium, the \% cells grown in 9-cis-RA was not significantly affected, although the neurite extension was significantly reduced. These data suggest a role for $\mathrm{Ca}^{2+}$ influx in the 9-cis-RA mediated neurite extension. Further experimentation will however be re- quired to fully elucidate the pathways involved in the RA neuriteinducing effects, as well as to determine the potential role of the nuclear receptors in this effect.

RA acts as a transcriptional activator of many downstream regulatory molecules by binding to specific nuclear receptors, the retinoic acid receptors (RARs) and the retinoid X receptors (RXRs). In nonchordates (including Lymnaea) an RAR has not yet been identified (Wiens et al., 2003), with the only invertebrate genes with any degree of similarity having been identified in Drosophila melanogaster (Oro et al., 1988) and the sponge, Geodia cydonium (Biesalski et al., 1992). However, C. Carter, R. Carlone, J. Dmetrichuk, and G. Spencer (GenBank Accession No. AY846875, unpublished data) have cloned a full-length cDNA for RXR from the Lymnaea CNS which has $89 \%$ identity in the DNA binding domain and $81 \%$ identity in the ligand-binding domain with rat $\operatorname{RXR} \alpha$. Although it is not yet known whether 9-cis-RA binds to the RXR in Lymnaea, when the RXR from the mollusc Biomphalaria glabrata was transfected into mammalian cell lines, it showed transactivated transcription of a reporter gene in the presence of 9-cis-RA (Bouton et al., 2005). This suggests that the molluscan RXR has the capability to bind 9-cis-RA, activate transcription and may play a dominant role in invertebrate retinoid signaling. Interestingly, Nowickyj et al. (2008) showed that the locust RXR binds both 9-cis and atRA isomers with similar affinity, and at a level 2 magnitudes higher than DHA (docosahexaenoic acid, a putative nonretinoid RXR agonist), suggesting it is a retinoid-specific receptor. atRA has also been shown to upregulate RXR expression in the marine sponge in addition to exerting morphogenetic effects (Wiens et al., 2003). Although it is generally believed that the vertebrate RXRs only bind 9-cis-RA (Chambon, 1996), the above data and previous studies strongly suggest that the invertebrate RXRs might also bind atRA with a similar affinity to 9-cis-RA. Due to the similar physiological effects of the two isomers in our study, we think it likely that atRA and 9-cis RA may be acting via the same mechanism, although we have not yet determined whether this mechanism involves the LymRXR, nor have we examined the binding affinities of the LymRXR.

In summary, these results represent the first direct evidence of endogenous retinoids (by their specific configuration) in the invertebrate CNS and provide evidence for a similar physiological role for both atRA and 9-cis-RA in the regeneration and growth cone guidance of cultured neurons, at least in this invertebrate species.

\section{References}

Barua AB, Furr HC (1998) Properties of retinoids. Structure, handling, and preparation. Methods Mol Biol 89:3-28.

Biesalski HK, Doepner G, Tzimas G, Gamulin V, Schröder HC, Batel R, Nau H, Müller WE (1992) Modulation of myb gene expression in sponges by retinoic acid. Oncogene 7:1765-1774.

Bouton D, Escriva H, deMendonca R, Glineur C, Bertin B, Noel C, RobinsonRechavi M, de Groot A, Cornette J, Laudet V, Pierce R (2005) A conserved retinoid X receptor (RXR) from the mollusk Biomphalaria glabrata transactivates transcription in the presence of retinoids. J Mol Endocrin 34:567-582.

Brodeur H, Parisotto M, Chagnon S, Mader S, Bhat PV (2007) Isomerspecific retinoic acid biosynthesis in HeLa cells expressing recombinant class I aldehyde dehydrogenases. Biochim Biophys Acta 1770:1548-1556.

Chambon P (1996) A decade of molecular biology of retinoic acid receptors. FASEB J 10:940-954.

Corcoran J, Shroot B, Pizzey J, Maden M (2000) The role of retinoic acid receptors in neurite outgrowth from different populations of embryonic mouse dorsal root ganglia. J Cell Sci 113:2567-2574. 
Créton R, Zwaan G, Dohmen R (1993) Specific developmental defects in molluscs after treatment with retinoic acid during gastrulation. Dev Grow Diff 35:357-364.

Dalfó D, Albalat R, Molotkov A, Duester G, Gonzàlez-Duarte R (2002) Retinoic acid synthesis in the prevertebrate amphioxus involves retinol oxidation. Dev Genes Evol 212:388-393.

Dmetrichuk JM, Spencer GE, Carlone RL (2005) Retinoic acid-dependent attraction of adult spinal cord axons towards regenerating newt limb blastemas in vitro. Dev Biol 281:112-120.

Dmetrichuk JM, Carlone RL, Spencer GE (2006) Retinoic acid induces neurite outgrowth and growth cone turning in invertebrate neurons. Dev Biol 294:39-49.

Driessen CA, Winkens HJ, Kuhlmann ED, Janssen AP, Van Vugt AH, Deutman AF, Janssen JJ (1998) The visual cycle retinol dehydrogenase: possible involvement in the 9-cis retinoic acid biosynthetic pathway. FEBS Lett 428:135-140.

Eakin RM, Brandenburger JL (1968) Localization of vitamin A in the eye of a pulmonate snail. Proc Natl Acad Sci U S A 60:140-145.

Feng ZP, Hasan SU, Lukowiak K, Syed NI (2000) Target cell contact suppresses neurite outgrowth from soma-soma paired Lymnaea neurons. J. Neurobiol 42:357-369.

Gu PL, Gunawardene YI, Chow BC, He JG, Chan SM (2002) Characterization of a novel cellular retinoic acid/retinol binding protein from shrimp: expression of the recombinant protein for immunohistochemical detection and binding assay. Gene 288:77-84.

Han G, Chang B, Connor MJ, Sidell N (1995) Enhanced potency of 9-cis versus all-trans-retinoic acid to induce the differentiation of human neuroblastoma cells. Differentiation 59:61-69.

Haskell BE, Stach RW, Werrbach-Perez K, Perez-Polo JR (1987) Effect of retinoic acid on nerve growth factor receptors. Cell Tissue Res 247:67-73

Hellemans K, Grinko I, Rombouts K, Schuppan D, Geerts A (1999) Alltrans and 9-cis retinoic acid alter rat hepatic stellate cell phenotype differentially. Gut 45:134-142.

Hoover F, Gundersen TE, Ulven SM, Michaille JJ, Blanchet S, Blomhoff R, Glover JC (2001) Quantitative assessment of retinoid signaling pathways in the developing eye and retina of the chicken embryo. J Comp Neurol 436:324-335.

Hopkins P (2001) Limb regeneration in the fiddler crab, Uca pugilator: hormonal and growth factor control. Am Zool 41:389-398.

Horton C, Maden M (1995) Endogenous distribution of retinoids during normal development and teratogenesis in the mouse embryo. Dev Dyn 202:312-323.

Kane M, Chen N, Sparks S, Napoli J (2005) Quantification of endogenous retinoic acid in limited biological samples by LC/MS/MS. Biochem J 388:363-369.

Kater SB, Mills LR (1991) Regulation of growth cone behavior by calcium. J Neurosci 11:891-899.

Kraft JC, Juchau MR (1993) 9-cis-retinoic acid: a direct-acting dysmorphogen. Biochem Pharmacol 46:709-716.

Kraft JC, Schuh T, Juchau M, Kimelman D (1994) The retinoid X receptor ligand, 9-cis-retinoic acid, is a potential regulator of early Xenopus development. Proc Natl Acad Sci U S A 91:3067-3071.

Labrecque J, Dumas F, Lacroix A, Bhat PV (1995) A novel isozyme of aldehyde dehydrogenase specifically involved in the biosynthesis of 9-cis and all-trans retinoic acid. Biochem J 305:681-684.

Lovat PE, Irving H, Malcolm AJ, Pearson AD, Redfern CP (1997) 9-cis retinoic acid-a better retinoid for the modulation of differentiation, proliferation and gene expression in human neuroblastoma. J Neurooncol 31:85-91.

Maden M (2007) Retinoic acid in the development, regeneration and maintenance of the nervous system. Nature Rev Neurosci 8:755-765.

Maden M, Hind M (2003) Retinoic acid, a regeneration-inducing molecule. Dev Dyn 226:237-244.

Maden M, Keen G, Jones GE (1998a) Retinoic acid as a chemotactic molecule in neuronal development. Int J Dev Neurosci 16:317-322.

Maden M, Sonneveld E, van der Saang PT, Gale E (1998b) The distribution of endogenous retinoic acid in the chick embryo: implications for developmental mechanisms. Development 125:4133-4144.

Malik MA, Blusztajn JK, Greenwood CE (2000) Nutrients as neurotrophic factors in the central nervous system: Role of retinoic acid. J Nutr Biochem 11:2-13.
Mansfield SG, Cammer S, Alexander SC, Muehleisen DP, Gray RS, Tropsha A, Bollenbacher WE (1998) Molecular cloning and characterization of an invertebrate cellular RA binding protein. Proc Natl Acad Sci U S A 95:6825-6830.

McCaffery P, Evans J, Koul O, Volpert A, Reid K, Ullman MD (2002) Retinoid quantification by HPLC/MS(n). J Lipid Res 43:1143-1149.

McFarlane S, Holt C (1997) Growth factors: a role in guiding axons? Trends in Cell Biol 7:424-430.

Mey J, Rombach N (1999) RA increases BDNF-dependent regeneration of chick retinal ganglion cells in vitro. Neuroreport 10:3573-3577.

Munno DW, Woodin MA, Lukowiak K, Syed NI, Dickinson PS (2000) Different extrinsic trophic factors regulate neurite outgrowth and synapse formation between identified Lymnaea neurons. J Neurobiol 44:20-30.

Nowickyj SM, Chithalen JV, Cameron D, Tyshenko MG, Petkovich M, Wyatt GR, Jones G, Walker VK (2008) Locust retinoid X receptors: 9-cisretinoic acid in embryos from a primitive insect. Proc Natl Acad Sci U S A 105:9540-9545.

Oro AE, Ong ES, Margolis JS, Posakony JW, McKeown M, Evans RM (1988) The Drosophila gene knirps-related is a member of the steroid-receptor gene superfamily. Nature 336:493-496.

Paik J, Blaner WS, Swisshelm K (2005) Cis-retinol dehydrogenase: 9-cisretinol metabolism and its effect on proliferation of human MCF7 breast cancer cells. Exp Cell Res 303:183-196.

Pedersen WA, Berse B, Schüler U, Wainer BH, Blusztajn JK (1995) Alltrans- and 9-cis-retinoic acid enhance the cholinergic properties of a murine septal cell line: evidence that the effects are mediated by activation of retinoic acid receptor-alpha. J Neurochem 65:50-58.

Plum LA, Clagett-Dame M (1995) 9-cis-retinoic acid selectively activates the cellular retinoic acid binding protein-II gene in human neuroblastoma cells. Arch Biochem Biophys 319:457-463.

Plum LA, Parada LF, Tsoulfas P, Clagett-Dame M (2001) Retinoic acid combined with neurotrophin-3 enhances the survival and neurite outgrowth of embryonic sympathetic neurons. Exp Biol Med (Maywood) 226:766-775.

Prince DJ, Carlone R (2003) Retinoic acid involvement in the reciprocal neurotrophic interactions between newt spinal cord and limb blastemas in vitro. Dev Brain Res 140:67-73.

Ridgway RL, Syed NI, Lukowiak K, Bulloch AG (1991) Nerve growth factor (NGF) induces sprouting of specific neurons of the snail Lymnaea stagnalis. J Neurobiol 22:377-390.

Romert A, Tuvendal P, Simon A, Dencker L, Eriksson U (1998) The identification of a 9-cis retinol dehydrogenase in the mouse embryo reveals a pathway for synthesis of 9-cis retinoic acid. Proc Natl Acad Sci U S A 95:4404-4409.

Scadding SR, Maden M (1994) Retinoic acid gradients during limb regeneration. Dev Biol 162:608-617.

Schmidt CK, Volland J, Hamscher G, Nau H (2002) Characterization of a new endogenous vitamin A metabolite. Biochim Biophys Acta 1583:237-251.

Shirley MA, Bennani YL, Boehm MF, Breau AP, Pathirana C, Ulm EH (1996) Oxidative and reductive metabolism of 9-cis-retinoic acid in the rat. Identification of 13,14-dihydro-9-cis-retinoic acid and its taurine conjugate. Drug Metab Dispos 24:293-302.

Spencer GE, Lukowiak K, Syed NI (1996) Dopamine regulation of neurite outgrowth from identified Lymnaea neurons in culture. Cell Mol Neurobiol 16:577-589.

Spencer GE, Lukowiak K, SyedI N (2000) Transmitter-receptor interaction between growth cones of identified Lymnaea neurons determine target cell selection in vitro. J Neurosci 20:8077-8086.

Syed NI, Bulloch AG, Lukowiak K (1990) In vitro reconstruction of the respiratory central pattern generator of the mollusc Lymnaea stagnalis. Science 250:282-285.

Thaller C, Eichele G (1987) Identification and spatial distribution of retinoids in the developing chick limb bud. Nature 327:625-628.

Thaller C, Hofmann C, Eichele G (1993) 9-cis-retinoic acid, a potent inducer of digit pattern duplications in the chick wing bud. Development 118:957-965.

Ulven SM, Gundersen TE, Sakhi AK, Glover JC, Blomhoff R (2001) Quantitative axial profiles of retinoic acid in the embryonic mouse spinal cord: 9-cis retinoic acid only detected after all-trans-retinoic acid levels are super-elevated experimentally. Dev Dyn 222:341-353. 
Viviano CM, Horton C, Maden M, Brockes JP (1995) Synthesis and release of 9 -cis retinoic acid by the urodele wound epidermis. Development 121:3753-3762.

Wagner M (1998) Detection and measurement of retinoic acid production by isolated tissues using retinoic acid-sensitive reporter cell lines. Methods Mol Biol 89:41-53.

Wagner M, Han B, Jessell TM (1992) Regional differences in retinoid release from embryonic neural tissue detected by an in vitro reporter assay. Development 116:55-66.

Werner EA, Deluca HF (2002) Retinoic acid is detected at relatively high levels in the CNS of adult rats. Am J Physiol Endocrinol Metab 282:E672-E678.

Wiens M, Batel R, Korzhev M, Muller WE (2003) Retinoid X receptor and
RA response in the marine sponge Suberites domuncula. J Exp Biol 206:3261-3271.

Wong RG, Hadley RD, Kater SB, Hauser GC (1981) Neurite outgrowth in molluscan organ and cell cultures: the role of conditioning factor(s). J Neurosci 1:1008-1021.

Wuarin L, Sidell N, de Vellis J (1990) Retinoids increase perinatal spinal cord neuronal survival and astroglial differentiation. Int J Dev Neurosci 8:317-326.

Xu J, Drew PD (2006) 9-cis retinoic acid suppresses inflammatory responses of microglia and astrocytes. J Neuroimmunology 171:135-144.

Yamamoto M, McCaffery P, Drager UC (1996) Influence of the choroids plexus on cerebellar development: analysis of retinoic acid synthesis. Dev Brain Res 93:182-190. 